\title{
Effective Tolman temperature induced by trace anomaly
}

\author{
Myungseok Eune ${ }^{1, \mathrm{a}}$, Yongwan Gim ${ }^{2,3, \mathrm{~b}}$, Wontae $\mathrm{Kim}^{2, \mathrm{c}}$ \\ ${ }^{1}$ Department of Civil Engineering, Sangmyung University, Cheonan 31066, Republic of Korea \\ ${ }^{2}$ Department of Physics, Sogang University, Seoul 04107, Republic of Korea \\ ${ }^{3}$ Research Institute for Basic Science, Sogang University, Seoul, 04107, Republic of Korea
}

Received: 20 January 2017 / Accepted: 7 April 2017 / Published online: 17 April 2017

(C) The Author(s) 2017. This article is an open access publication

\begin{abstract}
Despite the finiteness of stress tensor for a scalar field on the four-dimensional Schwarzschild black hole in the Israel-Hartle-Hawking vacuum, the Tolman temperature in thermal equilibrium is certainly divergent on the horizon due to the infinite blue-shift of the Hawking temperature. The origin of this conflict is due to the fact that the conventional Tolman temperature was based on the assumption of a traceless stress tensor, which is, however, incompatible with the presence of the trace anomaly responsible for the Hawking radiation. Here, we present an effective Tolman temperature which is compatible with the presence of the trace anomaly by using the modified Stefan-Boltzmann law. Eventually, the effective Tolman temperature turns out to be finite everywhere outside the horizon, and so an infinite blue-shift of the Hawking temperature at the event horizon does not appear any more. In particular, it is vanishing on the horizon, so that the equivalence principle is exactly recovered at the horizon.
\end{abstract}

\section{Introduction}

A quantum black hole $[1,2]$ in the Israel-Hartle-Hawking vacuum $[3,4]$ could be characterized by the Hawking temperature $T_{\mathrm{H}}$ which is given by the surface gravity. The local temperature in a proper frame as the Tolman temperature can be defined in the form of the blue-shifted Hawking temperature as $[5,6]$

$T_{\mathrm{loc}}=\frac{T_{\mathrm{H}}}{\sqrt{-g_{00}(r)}}$,

which is infinite at the horizon due to the infinite blue-shift of the Hawking temperature, though it reduces to the Hawking temperature at infinity.

\footnotetext{
a e-mail: eunems@smu.ac.kr

b e-mail: yongwan89@sogang.ac.kr

c e-mail:wtkim@sogang.ac.kr
}

On the other hand, the renormalized stress tensor for a conformal scalar field could be finite on the background of the Schwarzschild black hole [7]. At infinity, the proper energy density $\rho$ is positive finite, which is consistent with the Stefan-Boltzmann law as $\rho=\sigma T_{\mathrm{H}}^{4}$, where $\sigma=\pi^{2} / 30$. If one considered a motion of an inertial observer [7-10], the negative proper energy density could be found near the horizon in various vacua and its role was also discussed in connection with the information loss paradox [10]. However, it might be interesting to note that the local temperature (1) is infinite at the horizon, although the proper energy density at the horizon $r_{\mathrm{H}}$ is negative finite as $\rho\left(r_{\mathrm{H}}\right)=-12 \sigma T_{\mathrm{H}}^{4}$ as seen in Ref. [7].

Now, it appears to be puzzling in that the Tolman temperature at the horizon is positively divergent despite the negative finite energy density there. More worse, the energy density happens to vanish at a certain point outside the horizon [7], but the local temperature (1) is positive finite at that point. In these regards, the Tolman temperature runs contrary to the finite renormalized stress tensor, which certainly requires that the Stefan-Boltzmann law to relate the stress tensor to the proper temperature should be appropriately modified in such a way that they are compatible each other.

To resolve the above conflict between the finiteness of the renormalized stress tensor and the divergent behavior of the proper temperature, it is worth noting that the usual Tolman temperature rests upon the traceless stress tensor; however, the trace of the renormalized stress tensor is actually not traceless because of the trace anomaly. So we should find a modified Stefan-Boltzmann law in order to get the proper temperature commensurate with the finite renormalized stress tensor. In fact, this was successfully realized in the two-dimensional case where the stress tensor was perfect fluid [11]. In this work, we would like to extend the above issue to the case of the four-dimensional more realistic Schwarzschild black hole, where the renormalized stress tensor is no more isotropic. 
Using the exact thermal stress tensor calculated in Ref. [7], we solve the covariant conservation law and the equation for the trace anomaly, and then obtain the proper quantities such as the proper energy density and pressures written explicitly in terms of the trace anomaly in Sect. 2. In Sect. 3, we derive the effective Tolman temperature from the modified StefanBoltzmann law based on thermodynamic analysis. It shows that the effective Tolman temperature exactly reproduces the Hawking temperature at infinity, but it has a maximum at a finite distance outside the horizon and eventually it is vanishing rather than divergent on the horizon. Finally, a conclusion and a discussion are given in Sect. 4.

\section{Proper quantities in terms of trace anomaly}

We start with a four-dimensional Schwarzschild black hole governed by the static line element as

$\mathrm{d} s^{2}=-f(r) \mathrm{d} t^{2}+\frac{1}{f(r)} \mathrm{d} r^{2}+r^{2}\left(\mathrm{~d} \theta^{2}+\sin ^{2} \theta \mathrm{d} \phi^{2}\right)$,

where the metric function is $f(r)=1-2 G M / r$. The renormalized stress tensor for a conformal scalar field on the Schwarzschild black hole was obtained in the Israel-HartleHawking vacuum $[3,4]$ by using the Gaussian approximation [7]

$$
\begin{aligned}
T_{v}^{\mu}= & \frac{\pi^{2}}{90}\left(\frac{1}{8 \pi M}\right)^{4}\left[\frac{1-\left(4-\frac{6 M}{r}\right)^{2}\left(\frac{2 M}{r}\right)^{6}}{\left(1-\frac{2 M}{r}\right)^{2}}\left(\delta_{v}^{\mu}-4 \delta_{0}^{\mu} \delta_{v}^{0}\right)\right. \\
& \left.+24\left(\frac{2 M}{r}\right)^{6}\left(3 \delta_{0}^{\mu} \delta_{v}^{0}+\delta_{1}^{\mu} \delta_{v}^{1}\right)\right]
\end{aligned}
$$

where it is finite everywhere.

On general grounds, the trace anomaly can be written in the form of curvature invariants as

$T_{\mu}^{\mu}=\alpha\left(\mathcal{F}+\frac{2}{3} \square R\right)+\beta \mathcal{G}$,

where $\mathcal{F}=R^{\mu \nu \rho \sigma} R_{\mu \nu \rho \sigma}-2 R^{\mu \nu} R_{\mu \nu}+R^{2} / 3$ and $\mathcal{G}=$ $R^{\mu \nu \rho \sigma} R_{\mu \nu \rho \sigma}-4 R^{\mu \nu} R_{\mu \nu}+R^{2}$ [12-16]. There have been a lot of applications of trace anomalies to Hawking radiation and black hole thermodynamics in wide variety of cases of interest [17-28]. The coefficients $\alpha$ and $\beta$ are related to the number of conformal fields such as real scalar fields $N_{\mathrm{S}}$, Dirac (fermion) fields $N_{\mathrm{F}}$, and vector fields $N_{\mathrm{V}}$, such that they are fixed as $\alpha=\left(120(4 \pi)^{2}\right)^{-1}\left(N_{\mathrm{S}}+6 N_{\mathrm{F}}+12 N_{\mathrm{V}}\right)$ and $\beta=-\left(360(4 \pi)^{2}\right)^{-1}\left(N_{\mathrm{S}}+11 N_{\mathrm{F}}+62 N_{\mathrm{V}}\right)$. For the Ricci flat spacetime with a single conformal scalar field, the trace anomaly reduces to

$T_{\mu}^{\mu}=\frac{1}{2880 \pi^{2}} R^{\mu \nu \rho \sigma} R_{\mu \nu \rho \sigma}=\frac{M^{2}}{60 \pi^{2} r^{6}}$,

and then the trace for the stress tensor (3) is exactly in accord with the conformal anomaly (5).

In contrast to the two-dimensional case [11], the stress tensor appears anisotropic in the spherically symmetric black hole in four dimensions, and so the form of the stress tensor (3) should be generically written as $[29,30]$

$T^{\mu \nu}=\left(\rho+p_{t}\right) u^{\mu} u^{\nu}+p_{t} g^{\mu \nu}+\left(p_{r}-p_{t}\right) n_{(r)}^{\mu} n_{(r)}^{v}$.

The proper velocity $u^{\mu}$ is a timelike unit vector satisfying $u^{\mu} u_{\mu}=-1, n_{(r)}^{\mu}$ is the unit spacelike vector in the radial direction, and $n_{(\theta)}^{\mu}$ and $n_{(\phi)}^{\mu}$ are the unit normal vectors, which are orthogonal to $n_{(r)}^{\mu}$, satisfying $g_{\mu \nu} n_{(i)}^{\mu} n_{(j)}^{\mu}=\delta_{i j}$ and $n_{(i)}^{\mu} u_{\mu}=0$ where $i, j=r, \theta, \phi$. Thus the spacelike unit normal vectors are determined as

$n_{(r)}^{\mu}=(0, \sqrt{f(r)}, 0,0), \quad n_{(\theta)}^{\mu}=\left(0,0, \frac{1}{r}, 0\right)$,

$n_{(\phi)}^{\mu}=\left(0,0,0, \frac{1}{r \sin \theta}\right)$,

with the proper velocity

$u^{\mu}=\left(\frac{1}{\sqrt{f(r)}}, 0,0,0\right)$

for the frame dropped from rest. Then, from Eqs. (3), (6), (7), and (8), the proper energy density and pressures can be explicitly calculated by using the following relations:

$\rho=T_{\mu \nu} u^{\mu} u^{\nu}, \quad p_{r}=T_{\mu \nu} n_{(r)}^{\mu} n_{(r)}^{\nu}$,

$p_{t}=T_{\mu \nu} n_{(\theta)}^{\mu} n_{(\theta)}^{\nu}=T_{\mu \nu} n_{(\phi)}^{\mu} n_{(\phi)}^{\nu}$,

where the proper flux along $x^{i}$-direction can also be obtained by using the relation $\mathcal{F}_{i}=-T_{\mu \nu} u^{\mu} n_{(i)}^{\nu}$ but it trivially vanishes in thermal equilibrium [3,4].

Note that the energy density and pressures are not independent, as seen from the trace relation,

$T_{\mu}^{\mu}=-\rho+p_{r}+2 p_{t}$.

From Eqs. (3), (5), and (9), we find the additional relation

$p_{r}-p_{t}=\frac{1}{4} T_{\mu}^{\mu}$,

which characterizes the anisotropy between the tangential pressure and radial pressure. 
Let us now express the proper energy density and pressures formally in terms of the trace anomaly for our purpose. From Eq. (6), the covariant conservation law for the energymomentum tensor is rewritten as

$\partial_{r} p_{r}+\frac{2}{r}\left(p_{r}-p_{t}\right)+\frac{1}{2 f} \partial_{r} f\left(p_{r}+\rho\right)=0$.

Plugging Eqs. (10) and (11) into Eq. (12), one can obtain the simplified form of

$\partial_{r} p_{r}+\frac{\partial_{r} f}{2 f} p_{r}=-\left(\frac{1}{2 r}+\frac{3 \partial_{r} f}{4 f}\right) T_{\mu}^{\mu}$,

which can be solved as

$p_{r}=\frac{1}{f^{2}}\left(C_{0}+\int^{r} \frac{f}{4 r}\left(-2 f+3 r \partial_{r} f\right) T_{\mu}^{\mu} \mathrm{d} r\right)$,

where $C_{0}$ is an integration constant. Additionally, from Eqs. (10) and (11), the tangential pressure and energy density can also be obtained:

$$
\begin{aligned}
p_{t} & =\frac{1}{f^{2}}\left(C_{0}-\frac{f^{2}}{4} T_{\mu}^{\mu}+\int^{r} \frac{f}{4 r}\left(-2 f+3 r \partial_{r} f\right) T_{\mu}^{\mu} \mathrm{d} r\right), \\
\rho & =\frac{3}{f^{2}}\left(C_{0}-\frac{f^{2}}{2} T_{\mu}^{\mu}+\int^{r} \frac{f}{4 r}\left(-2 f+3 r \partial_{r} f\right) T_{\mu}^{\mu} \mathrm{d} r\right) .
\end{aligned}
$$

The above proper quantities defined in freely falling frames were related to the trace anomaly conveniently, which will be used in the next section.

\section{Effective Tolman temperature}

In this section, we derive the proper temperature for the background of the four-dimensional Schwarzschild black hole based on the modified Stefan-Boltzmann law. First of all, we note that the volume of the system in the radial proper frame can be changed only along the radial direction on the spherically symmetric black hole, and thus obtain the thermodynamic first law written as

$\mathrm{d} U=T \mathrm{~d} S-p_{r} \mathrm{~d} V$

without recourse to the tangential work. From Eq. (17), one can immediately get

$\left(\frac{\partial U}{\partial V}\right)_{T}=T\left(\frac{\partial S}{\partial V}\right)_{T}-p_{r}$ and then, from the Maxwell relations such as $(\partial S / \partial V)_{T}=$ $\left(\partial p_{r} / \partial T\right)_{V}$, we obtain

$\rho=T\left(\frac{\partial p_{r}}{\partial T}\right)_{V}-p_{r}$

Using the fact that the trace anomaly is independent of temperature as $\partial_{T} T_{\mu}^{\mu}=0$ [31], from Eqs. (10) and (11), we also obtain

$$
\left(\frac{\partial \rho}{\partial T}\right)_{V}=\left(\frac{\partial p_{r}}{\partial T}\right)_{V}+2\left(\frac{\partial p_{t}}{\partial T}\right)_{V}
$$

and

$$
\left(\frac{\partial p_{r}}{\partial T}\right)_{V}=\left(\frac{\partial p_{t}}{\partial T}\right)_{V} .
$$

Plugging Eqs. (20) and (21) into Eq. (19), we get

$T\left(\frac{\partial \rho}{\partial T}\right)_{V}-4 \rho=\frac{3}{2} T_{\mu}^{\mu}$,

which is solved as

$\rho=3 \gamma T^{4}-\frac{3}{8} T_{\mu}^{\mu}$.

From Eqs. (10) and (11), the radial and tangential pressure are also derived as

$p_{r}=\gamma T^{4}+\frac{3}{8} T_{\mu}^{\mu}$,
$p_{t}=\gamma T^{4}+\frac{1}{8} T_{\mu}^{\mu}$,

respectively. The integration constant $\gamma$ is related to the Stefan-Boltzmann constant $\sigma$ as $\gamma=\sigma / 3=\pi^{2} / 90$ for a conformal scalar field [32]. For the traceless case, the modified Stefan-Boltzmann law (23) simply reduces to the usual one. The proper energy density in Eq. (23) is not necessarily positive definite thanks to the trace anomaly, so that the negative energy states are naturally permitted in this extended setting.

From Eqs. (23), (24), and (25), the proper temperature is obtained as

$$
\begin{aligned}
T & =\left[\frac{1}{\gamma}\left(p_{r}-\frac{3}{8} T_{\mu}^{\mu}\right)\right]^{1 / 4}=\left[\frac{1}{\gamma}\left(p_{t}-\frac{1}{8} T_{\mu}^{\mu}\right)\right]^{1 / 4} \\
& =\left[\frac{1}{3 \gamma}\left(\rho+\frac{3}{8} T_{\mu}^{\mu}\right)\right]^{1 / 4},
\end{aligned}
$$

and it can be compactly written in terms of the trace anomaly as 
$T=\frac{1}{\gamma^{1 / 4} \sqrt{f}}\left(C_{0}-\frac{3}{8} f^{2} T_{\mu}^{\mu}+\int^{r} \frac{f}{4 r}\left(-2 f+3 r \partial_{r} f\right) T_{\mu}^{\mu} \mathrm{d} r\right)^{1 / 4}$,

where we used Eqs. (14), (15), and (16). In the absence of the trace anomaly, the proper temperature (27) reduces to the usual Tolman temperature $[5,6]$. Requiring that the proper temperature (27) be coincident with the Hawking temperature $T_{\mathrm{H}}$ at infinity, we can fix the constant as $C_{0}=\gamma^{1 / 4} T_{\mathrm{H}}$.

Finally, plugging the trace anomaly (5) into Eq. (27), we obtain

$$
\begin{aligned}
T= & \frac{1}{8 \pi M \sqrt{f(r)}}\left[1-28\left(\frac{2 M}{r}\right)^{6}+48\left(\frac{2 M}{r}\right)^{7}\right. \\
& \left.-21\left(\frac{2 M}{r}\right)^{8}\right]^{1 / 4},
\end{aligned}
$$

which can be neatly factorized as

$$
\begin{aligned}
T=\frac{1}{8 \pi M \sqrt{f(r)}}[ & \left(1-\frac{2 M}{r}\right)^{2}\left(1+2\left(\frac{2 M}{r}\right)+3\left(\frac{2 M}{r}\right)^{2}\right. \\
& +4\left(\frac{2 M}{r}\right)^{3}+5\left(\frac{2 M}{r}\right)^{4}+6\left(\frac{2 M}{r}\right)^{5} \\
& \left.\left.-21\left(\frac{2 M}{r}\right)^{6}\right)\right]^{1 / 4}
\end{aligned}
$$

It seems to be interesting to note that the blue-shift factor in the denominator related to the origin of the divergence at the horizon can be canceled out, so that the effective Tolman temperature is written as

$T=\frac{1}{8 \pi M}\left[\left(1-\frac{2 M}{r}\right) \sum_{n=1}^{6} \frac{n(n+1)}{2}\left(\frac{2 M}{r}\right)^{n-1}\right]^{1 / 4}$.

Thus the red-shift factor responsible for the infinite blue-shift of the Hawking temperature on the horizon does not appear any more in the effective Tolman temperature. As seen from Fig. 1, the behavior of the temperature (30) shows that it is finite everywhere and approaches the Hawking temperature at infinity. In particular, it is vanishing on the horizon, so that the freely falling observer from rest does not see any excited particles. On the contrary to the naively expected divergence from the usual Tolman temperature at the horizon, the high frequency quanta could not be found on the horizon, which would be compatible with the result that the equivalence principle could be recovered at the horizon [33].

The divergent dashed curve near the horizon in Fig. 1 could be made finite by taking into account the quantum effect via the trace anomaly, which is reminiscent of the vanishing

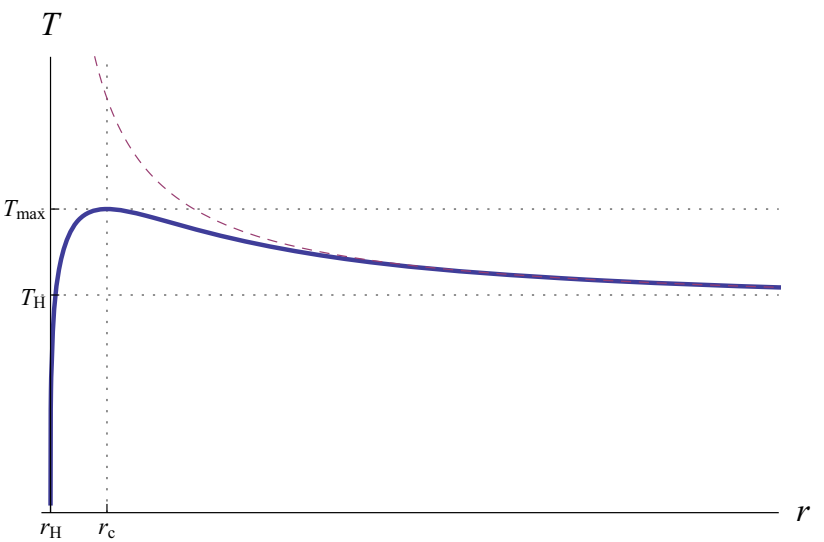

Fig. 1 The dashed curve shows the behavior of the usual Tolman temperature of being divergent on the horizon. The solid curve is the effective Tolman temperature, which is finite everywhere. In particular, it vanishes at the horizon and has a maximum $T_{\max } \sim 1.51 T_{\mathrm{H}}$ at $r_{c} \sim 1.31 r_{H}$. All the curves approach the Hawking temperature at infinity, whereas they are very different from each other near the horizon where quantum effects are significant

Hawking temperature in the noncommutative Schwarzschild black hole based on the different assumptions of quantization rules [34]. The proper temperature based on the effective temperature method is also compatible with the present result in the sense that the proper temperature vanishes at the horizon [35].

\section{Conclusion and discussion}

It has been widely believed that the Tolman temperature is divergent at the horizon due to the infinite blue-shift of the Hawking radiation. However, the usual Stefan-Boltzmann law assuming the traceless stress tensor should be consistently modified in order to discuss the case where the stress tensor is no longer traceless in the process of the Hawking radiation. From the modified Stefan-Boltzmann law, we obtained the effective Tolman temperature without the redshift factor related to the origin of the divergence at the horizon, so that it is finite everywhere outside the black hole horizon.

The intriguing behavior of the effective Tolman temperature on the horizon may be understood by the Unruh effect [36]. The static metric (2) near the horizon can be written by the Rindler metric for a large black hole whose curvature scale is negligible. The Unruh temperature is divergent due to the infinite acceleration of the frame where the fixed detector is very close to the horizon. So the Unruh temperature is equivalent to the locally fiducial temperature for the Schwarzschild black hole [33]. Conversely speaking, based on the equivalence principle, the Unruh temperature measured by the geodesic detector should vanish on the horizon 
since the proper acceleration of the geodesic detector vanishes. In this regard, it appears natural to conclude that the freely falling observer from rest does not see any excited particles on the horizon in thermal equilibrium and thus the effective Tolman temperature vanishes at the horizon.

On the other hand, AMPS argument is that the firewall on the horizon should be defined in an evaporating black hole rather than the black hole in thermal equilibrium [37]. The firewall is certainly characterized by the divergent proper temperature in that the average frequency $\omega$ of an excited particle with a thermal bath can be identified with the proper temperature as $\omega \sim T$. Using the advantage of the effective Tolman temperature, we find the reason why the firewall could not exist in thermal equilibrium: the fact that the redshift factor responsible for the divergence at the horizon could be canceled out.

Acknowledgements We would like to thank Jeong-Hyuck Park and Edwin J. Son for exciting discussions. W. Kim was supported by the National Research Foundation of Korea (NRF) grant funded by the Korea government (MSIP) (2017R1A2B2006159).

Open Access This article is distributed under the terms of the Creative Commons Attribution 4.0 International License (http://creativecomm ons.org/licenses/by/4.0/), which permits unrestricted use, distribution, and reproduction in any medium, provided you give appropriate credit to the original author(s) and the source, provide a link to the Creative Commons license, and indicate if changes were made. Funded by SCOAP ${ }^{3}$.

\section{References}

1. S.W. Hawking, Particle creation by black holes. Commun. Math. Phys. 43, 199-220 (1975)

2. S.W. Hawking, Breakdown of predictability in gravitational collapse. Phys. Rev. D 14, 2460-2473 (1976)

3. J.B. Hartle, S.W. Hawking, Path integral derivation of black hole radiance. Phys. Rev. D 13, 2188-2203 (1976)

4. W. Israel, Thermo field dynamics of black holes. Phys. Lett. A 57, 107-110 (1976)

5. R.C. Tolman, On the weight of heat and thermal equilibrium in general relativity. Phys. Rev. 35, 904-924 (1930)

6. R. Tolman, P. Ehrenfest, Temperature equilibrium in a static gravitational field. Phys. Rev. 36, 1791-1798 (1930)

7. D.N. Page, Thermal stress tensors in static Einstein spaces. Phys. Rev. D 25, 1499 (1982)

8. L.H. Ford, T.A. Roman, Motion of inertial observers through negative energy. Phys. Rev. D 48, 776-782 (1993). arXiv:gr-qc/9303038

9. L.H. Ford, Negative energy densities in quantum field theory. arXiv:0911.3597

10. B. Freivogel, Energy and information near black hole horizons. JCAP 1407, 041 (2014). arXiv: 1401.5340

11. Y. Gim, W. Kim, A. Quantal, Tolman Temperature. Eur. Phys. J. C 75, 549 (2015). arXiv: 1508.00312

12. S. Deser, M.J. Duff, C.J. Isham, Nonlocal conformal anomalies. Nucl. Phys. B 111, 45 (1976)

13. M.J. Duff, Observations on conformal anomalies. Nucl. Phys. B 125, 334 (1977)
14. N.D. Birrell, P.C.W. Davies, Quantum Fields in Curved Space. Cambridge Monographs on Mathematical Physics (Cambridge University Press, Cambridge, 1984)

15. S. Deser, A. Schwimmer, Geometric classification of conformal anomalies in arbitrary dimensions. Phys. Lett. B 309, 279-284 (1993). arXiv:hep-th/9302047

16. M.J. Duff, Twenty years of the Weyl anomaly. Class. Quantum Gravity 11, 1387-1404 (1994). arXiv:hep-th/9308075

17. E. Elizalde, S.D. Odintsov, Renormalization of 2-D quantum gravity with matter in connection with black holes. JETP Lett. 56, 185187 (1992)

18. E. Elizalde, S.D. Odintsov, One loop renormalization in twodimensional matter dilaton quantum gravity and charged black holes. Nucl. Phys. B 399, 581-600 (1993). arXiv:hep-th/9207046

19. S. Nojiri, S.D. Odintsov, Effective action for conformal scalars and anti-evaporation of black holes. Int. J. Mod. Phys. A 14, 1293-1304 (1999). arXiv:hep-th/9802160

20. S. Nojiri, S.D. Odintsov, Quantum evolution of Schwarzschildde Sitter (Nariai) black holes. Phys. Rev. D 59, 044026 (1999). arXiv:hep-th/9804033

21. S. Nojiri, S.D. Odintsov, Quantum dilatonic gravity in $(\mathrm{D}=2)$ dimensions, $(\mathrm{D}=4)$-dimensions and $(\mathrm{D}=5)$-dimensions. Int. J. Mod. Phys. A 16, 1015-1108 (2001). arXiv:hep-th/0009202

22. E. Elizalde, S. Nojiri, S.D. Odintsov, Possible quantum instability of primordial black holes. Phys. Rev. D 59, 061501 (1999). arXiv:hep-th/9901026

23. A. Burinskii, E. Elizalde, S.R. Hildebrandt, G. Magli, Regular sources of the Kerr-Schild class for rotating and nonrotating black hole solutions. Phys. Rev. D 65, 064039 (2002). arXiv:gr-qc/0109085

24. R.-G. Cai, L.-M. Cao, N. Ohta, Black holes in gravity with conformal anomaly and logarithmic term in black hole entropy. JHEP 04, 082 (2010). arXiv:0911.4379

25. H. Kawai, Y. Yokokura, Phenomenological description of the interior of the Schwarzschild black hole. Int. J. Mod. Phys. A 30, 1550091 (2015). arXiv:1409.5784

26. A.R. Vieira, J.C.C. Felipe, G. Gazzola, M. Sampaio, One-loop conformal anomaly in an implicit momentum space regularization framework. Eur. Phys. J. C 75, 338 (2015). arXiv:1505.05319

27. H. Kawai, Y. Yokokura, Interior of black holes and information recovery. Phys. Rev. D 93, 044011 (2016). arXiv: 1509.08472

28. H. Kawai, Y. Yokokura, A model of black hole evaporation and 4D Weyl anomaly. arXiv: 1701.03455

29. S.W. Hawking, G.F.R. Ellis, The Large Scale Structure of Spacetime (Cambridge University Press, Cambridge, 1973)

30. M.N. Christiansen, T.K. Rasmussen, Classical and Quantum Gravity Research (Nova Science Publishers, New York, 2008)

31. H. Boschi-Filho, C.P. Natividade, Anomalies in curved space-time at finite temperature. Phys. Rev. D 46, 5458-5466 (1992)

32. S.M. Christensen, S.A. Fulling, Trace anomalies and the Hawking effect. Phys. Rev. D 15, 2088-2104 (1977)

33. D. Singleton, S. Wilburn, Hawking radiation, Unruh radiation and the equivalence principle. Phys. Rev. Lett. 107, 081102 (2011). arXiv: 1102.5564

34. P. Nicolini, Noncommutative black holes, the final appeal to quantum gravity: a review. Int. J. Mod. Phys. A 24, 1229-1308 (2009). arXiv:0807.1939

35. L.C. Barbado, C. Barcel, L.J. Garay, G. Jannes, Hawking versus Unruh effects, or the difficulty of slowly crossing a black hole horizon. JHEP 10, 161 (2016). arXiv:1608.02532

36. W.G. Unruh, Notes on black hole evaporation. Phys. Rev. D 14, $870(1976)$

37. A. Almheiri, D. Marolf, J. Polchinski, J. Sully, Black holes: complementarity or firewalls? JHEP 02, 062 (2013). arXiv:1207.3123 